\title{
DIFFERENTIABLE EQUISINGULARITY OF HOLOMORPHIC FOLIATIONS
}

\author{
ROGÉRIO MOL \& RUDY ROSAS
}

\begin{abstract}
We prove that a $C^{\infty}$ equivalence between germs holomorphic foliations at $\left(\mathbb{C}^{2}, 0\right)$ establishes a bijection between the sets of formal separatrices preserving equisingularity classes. As a consequence, if one of the foliations is of second type, so is the other and they are equisingular.
\end{abstract}

\section{INTRODUCTION}

A celebrated theorem of Zariski [12] asserts that two topological equivalent germs of curves at $\left(\mathbb{C}^{2}, 0\right)$ are necessarily equisingular, that is, their desingularization by blow-ups are combinatorially isomorphic. In [1], the authors prove the following analogous result for holomorphic foliations at $\left(\mathbb{C}^{2}, 0\right)$, valid for the generic class of generalized curve foliations:

Theorem A. Let $\mathcal{F}$ and $\mathcal{F}^{\prime}$ topologically equivalent germs of holomorphic foliations at $\left(\mathbb{C}^{2}, 0\right)$. Suppose that $\mathcal{F}$ is a generalized curve. Then $\mathcal{F}^{\prime}$ is also a generalized curve foliation. Besides, $\mathcal{F}$ and $\mathcal{F}^{\prime}$ have isomorphic desingularizations.

The proof of this theorem is based upon the following result, also proved in 11 :

Theorem B. Let $\mathcal{F}$ be a generalized curve foliation at $\left(\mathbb{C}^{2}, 0\right)$ and let $\operatorname{Sep}(\mathcal{F})$ be its set of separatrices. Then, the desingularization of $\operatorname{Sep}(\mathcal{F})$ is also the desingularization of $\mathcal{F}$.

In fact, if $\mathcal{F}$ is topologically equivalent to $\mathcal{F}^{\prime}$, we have that $\operatorname{Sep}(\mathcal{F})$ and $\operatorname{Sep}\left(\mathcal{F}^{\prime}\right)$ are also topological equivalent, since the separatrices of a generalized curve foliation are convergent. Therefore, Theorem A follows from Theorem B and Zariski's Theorem. In general, the validity of Theorem A outside the class of generalized curve foliations is a difficult open problem. Actually, such a result would imply the topological invariance of the algebraic multiplicity of a holomorphic foliation, which is also an open problem (see [8, 9, 10]). The desingularization of a germ of foliation $\mathcal{F}$ is closely related to the desingularization of its set of separatrices $\operatorname{Sep}(\mathcal{F})$ — including the purely formal ones - , although Theorem B is not always true. Another serious difficulty is the fact that the topological equivalence does not naturally map purely formal separatrices of $\mathcal{F}$ into purely formal separatrices of $\mathcal{F}^{\prime}$, as in the case of convergent separatrices.

If the equivalence between $\mathcal{F}$ and $\mathcal{F}^{\prime}$ is supposed to be $C^{\infty}$, a correspondence among formal separatrices of both foliations can be established. Let $\Phi$ be such a $C^{\infty}$ equivalence

\footnotetext{
${ }^{1} 2000$ Mathematics Subject Classification: $32 \mathrm{~S} 65$.

${ }^{2}$ Keywords. Holomorphic foliations, vector fields, invariant curves, equidesingularization.

${ }^{3}$ Work supported by MATH-AmSud Project CNRS/CAPES/Concytec. First author supported by Universal/CNPq. Second author supported by Vicerrectorado de Investigación de la Pontificia Universidad Católica del Perú.
} 
and consider its Taylor series $\hat{\Phi}$ as a real formal diffeomorphism of $\left(\mathbb{C}^{2}, 0\right)$. Let $S$ be a possibly formal separatrix of $\mathcal{F}$, which can be seen as a parametrized two-dimensional real formal surface at $\left(\mathbb{C}^{2}, 0\right)$. Then the formal composition $\hat{\Phi}(S)$ is a parametrized twodimensional real formal surface at $\left(\mathbb{C}^{2}, 0\right)$. In this setting, we have:

Theorem I. Let $\Phi$ be a $C^{\infty}$ equivalence between two germs $\mathcal{F}$ and $\mathcal{F}^{\prime}$ of singular holomorphic foliations at $\left(\mathbb{C}^{2}, 0\right)$. Let $S$ be a separatrix of $\mathcal{F}$, considered as a parametrized two-dimensional real formal surface at $\left(\mathbb{C}^{2}, 0\right)$. Then the following properties hold:

(1) The real formal surface $\hat{\Phi}(S)$ is a real formal reparametrization of some separatrix $S^{\prime}$ of $\mathcal{F}^{\prime}$, denoted by $S^{\prime}=\Phi_{*}(S)$.

(2) Let $\mathscr{S}$ be the reduced curve defined as the union of a finite collection $S_{1}, \ldots, S_{m}$ of separatrices of $\mathcal{F}$. Denote by $\mathscr{S}^{\prime}$ the reduced curve defined as the union of $\Phi_{*}\left(S_{1}\right), \ldots, \Phi_{*}\left(S_{m}\right)$. Then $\mathscr{S}$ and $\mathscr{S}^{\prime}$ are equisingular.

As a consequence of Theorem If if $\mathcal{F}$ and $\mathcal{F}^{\prime}$ are $C^{\infty}$ equivalent foliations, then the sets of separatrices $\operatorname{Sep}(\mathcal{F})$ and $\operatorname{Sep}\left(\mathcal{F}^{\prime}\right)$ have isomorphic desingularizations. Taking into account that the property described in Theorem B is valid for the larger class of second type foliations (see [6]), we obtain the following equidesingularization result for $C^{\infty}$ equivalent foliations:

Theorem II. Let $\mathcal{F}$ and $\mathcal{F}^{\prime}$ be two germs of holomorphic foliations at $\left(\mathbb{C}^{2}, 0\right)$ equivalent by a germ of $C^{\infty}$ diffeomorphism. If $\mathcal{F}$ is a foliation of second type, then $\mathcal{F}^{\prime}$ is of second type. Moreover, $\mathcal{F}$ and $\mathcal{F}^{\prime}$ are equisingular.

This paper is structured in the following way. In sections 2 and 3 we present basic definitions and some properties of second type foliations. Next, in sections 1 and 5 , we introduce the notion of characteristic curves for germs of holomorphic foliations. These are one-dimensional real curves intrinsically associated to separatrices — both convergent and formal. Characteristic curves are invariant by $C^{\infty}$ equivalences and enable us to establish a one to one correspondence among separatrices of two $C^{\infty}$ equivalent foliations. This is done in section 6. Next, in section 7, we introduce the concept of formal real equivalence of formal complex curves and we show that this notion implies equisingularity (Theorem 7.2). In section 8, we present the proof of Theorem [. Finally, in section 9, we accomplish the proof of Theorem 11 .

\section{Foliations, SEPARATRices AND DESingularization}

A germ of singular holomorphic foliation $\mathcal{F}$ at $\left(\mathbb{C}^{2}, 0\right)$ is the object defined by an equation of the form $\omega=0$, where $\omega$ is a 1 -form $\omega=P(u, v) d u+Q(u, v) d v$ - or, equivalently, by the orbits of the germ of holomorphic vector field $\mathbf{v}=-Q(u, v) \partial / \partial u+P(u, v) \partial / \partial v-$, where $P, Q \in \mathbb{C}\{u, v\}$ are relatively prime, defining what we call a reduced equation. Two reduced 1 -forms $\omega$ and $\tilde{\omega}$ define the same foliation if and only if $\omega=u \tilde{\omega}$ for some unity $u \in \mathbb{C}\{u, v\}$. In general, we can assume that a $1-$ form $\omega=P(u, v) d u+Q(u, v) d v$ defines a foliation by taking as reduced equation $\omega / R=0$, where $R=\operatorname{gcd}(P, Q)$.

A considerable amount of information on the local topology and dynamics of a foliation is given by their separatrices. A separatrix for a foliation $\mathcal{F}$ is an invariant formal irreducible curve. Algebraically, it is defined by an irreducible formal series $f \in \mathbb{C}[[u, v]]$, with $f(0,0)=$ 0 , satisfying

$$
\omega \wedge d f=f h d u \wedge d v
$$


for some formal series $h \in \mathbb{C}[[u, v]]$. If $f$ can be taken in $\mathbb{C}\{u, v\}$, the separatrix is said to be analytic or convergent. We denote by $\operatorname{Sep}(\mathcal{F})$ the set of separatrices of $\mathcal{F}$ at $0 \in \mathbb{C}^{2}$.

The singularity $0 \in \mathbb{C}^{2}$ for $\mathcal{F}$ is said to be simple if the linear part $\operatorname{Dv}(0)$ of a vector field $\mathbf{v}$ inducing $\mathcal{F}$ has eigenvalues $\lambda_{1}, \lambda_{2} \in \mathbb{C}$ meeting one of the following conditions:

Case 1: $\lambda_{1} \lambda_{2} \neq 0$ and $\lambda_{1} / \lambda_{2} \notin \mathbb{Q}^{+}$. We say that $0 \in \mathbb{C}^{2}$ is non-degenerate or complex hyperbolic. The set of separatrices $\operatorname{Sep}(\mathcal{F})$ is formed by two transversal branches, both of them analytic.

Case 2: $\lambda_{1} \neq 0$ and $\lambda_{2}=0$. This is called a saddle-node singularity, for which there are formal coordinates $(u, v)$ such that $\mathcal{F}$ is induced by

$$
\omega=v\left(1+\lambda u^{k}\right) d u+u^{k+1} d v
$$

where $\lambda \in \mathbb{C}$ and $k \in \mathbb{Z}_{>0}$. The curve $\{u=0\}$, corresponding to the tangent direction defined by the non-zero eigenvalue, defines an analytic separatrix, called strong, whereas $\{v=0\}$ is tangent to a possibly formal separatrix, called weak or central. The integer $k+1>1$ is called tangency index of $\mathcal{F}$ with respect to the weak separatrix, or simply weak index, and will be denoted by $\operatorname{Ind}_{0}^{w}(\mathcal{F})$.

A global foliation $\mathcal{G}$ on a holomorphic surface $M$ corresponds to the assignment, for $p \in M$, of compatible local foliations $\mathcal{G}_{p}$. For instance, a holomorphic 1 -form $\omega$ on $M$ defines a foliation $\mathcal{G}$ by taking $\mathcal{G}_{p}$ as the local foliation defined by the germification of $\omega$ at $p$. Let $\mathcal{F}$ be a local foliation at $\left(\mathbb{C}^{2}, 0\right)$ defined by the 1 -form $\omega$ and let $\pi:(M, E) \rightarrow\left(\mathbb{C}^{2}, 0\right)$ be a sequence of punctual blow-ups starting at $0 \in \mathbb{C}^{2}$. The pull-back $1-$ form $\pi^{*} \omega$ defines a foliation $\tilde{\mathcal{F}}=\pi^{*} \mathcal{F}$ with isolated singularities on $(M, E)$ called the strict transform of $\mathcal{F}$ by $\pi$. We have the definition:

Definition 2.1. Let $\mathcal{G}$ be a foliation on $(M, E)$, where $E$ is a normal crossings divisor. With respect to the pair $(\mathcal{G}, E)$, we say that $p \in E$ is

(1) a regular point, if there are local analytic coordinates $(u, v)$ at $p$ such that $E \subset$ $\{u v=0\}$ and $\mathcal{G}: d u=0$

(2) a simple singularity, if $p$ is a simple singularity for $\mathcal{G}$ and $E \subset \operatorname{Sep}_{p}(\mathcal{G})$.

This allows us to present the notion of reduction of singularities of a foliation with respect to a normal crossings divisor:

Definition 2.2. Let $\mathcal{G}$ be a foliation on $(M, E)$, where $E$ is a normal crossings divisor. We say that $(\mathcal{G}, E)$ is reduced or desingularized if all points $p \in E$ are either regular or simple singularities for the pair $(\mathcal{G}, E)$. A reduction of singularities or desingularization for a germ of foliation $\mathcal{F}$ at $\left(\mathbb{C}^{2}, 0\right)$ is a morphism $\pi:(M, E) \rightarrow\left(\mathbb{C}^{2}, 0\right)$, formed by a composition of punctual blow-ups, such that $\left(\pi^{*} \mathcal{F}, E\right)$ is reduced.

For a local foliation $\mathcal{F}$ at $\left(\mathbb{C}^{2}, 0\right)$, there always exists a reduction of singularities (see 111 and 11). Besides, there exists a minimal one, in the sense that it factorizes, by an additional sequence of blow-ups, any other reduction of singularities of $\mathcal{F}$. In the sequel, whenever we refer to a reduction of singularities, we mean a minimal one.

Let $\pi:(M, E) \rightarrow\left(\mathbb{C}^{2}, 0\right)$ be a reduction of singularities for $\mathcal{F}$ and denote $\tilde{\mathcal{F}}=\pi^{*} F$. The divisor $E=\pi^{-1}(0)$ is a finite union of components which are embedded projective lines, crossing normally at corners. The regular points of $D$ are called trace points. A component $D \subset E$ can be: 
(1) non-dicritical, if $D$ is $\tilde{\mathcal{F}}$-invariant. In this case, $D$ contains a finite number of simple singularities. Each trace singularity carries a separatrix transversal to $E$, whose projection by $\pi$ is a branch in $\operatorname{Sep}(\mathcal{F})$.

(2) dicritical, if $D$ is not $\tilde{\mathcal{F}}$-invariant. The definition of desingularization gives that $D$ may intersect only non-dicritical components and that $\tilde{\mathcal{F}}$ is everywhere transverse do $D$. The $\pi$-image of a local leaf of $\tilde{\mathcal{F}}$ at each trace point of $D$ belongs to $\operatorname{Sep}(\mathcal{F})$.

For each $B \in \operatorname{Sep}(\mathcal{F})$ we associate the trace point $\tau_{E}(B) \in E$ given by $\pi^{*} B \cap E$. We define $\operatorname{Sep}(D)=\left\{B \in \operatorname{Sep}(\mathcal{F}) ; \tau_{E}(B) \in D\right\}$ as the set of branches attached to the component $D \subset E$. We thus have a decomposition $\operatorname{Sep}(\mathcal{F})=\operatorname{Iso}(\mathcal{F}) \cup \operatorname{Dic}(\mathcal{F})$, where

$$
\operatorname{Iso}(\mathcal{F})=\bigcup_{D \text { non-dicritical }} \operatorname{Sep}(D) \quad \text { and } \quad \operatorname{Dic}(\mathcal{F})=\bigcup_{D \text { dicritical }} \operatorname{Sep}(D) .
$$

Separatrices in $\operatorname{Iso}(\mathcal{F})$, known as isolated, can be additionally classified in two types. A branch $B \in \operatorname{Iso}(\mathcal{F})$ is strong or of Briot and Bouquet type if either $\tau_{E}(B)$ is a nondegenerate singularity or if $\tau_{E}(B)$ is a saddle-node singularity with $\pi^{*} B$ as its strong separatrix. On the other hand, $B \in \operatorname{Iso}(\mathcal{F})$ is weak if $\tau_{E}(B)$ is a saddle-node singularity whose weak separatrix is $\pi^{*} B$. This classification engenders the decomposition $\operatorname{Iso}(\mathcal{F})=$ $\operatorname{Iso}^{s}(\mathcal{F}) \cup \operatorname{Iso}^{w}(\mathcal{F})$, where notations are self-evident. Note that $\operatorname{Iso}(\mathcal{F})$ is a finite set and all purely formal separatrices of $\mathcal{F}$ are contained in $\operatorname{Iso}^{w}(\mathcal{F})$.

On the other hand, if non-empty, $\operatorname{Dic}(\mathcal{F})$ is an infinite set of analytic separatrices, called dicritical. A foliation $\mathcal{F}$ may be classified either as non-dicritical - when $\operatorname{Sep}(\mathcal{F})$ is finite, which happens when $\operatorname{Dic}(\mathcal{F})=\emptyset$ - or as dicritical, otherwise.

Let $\mathcal{F}$ be a foliation at $\left(\mathbb{C}^{2}, 0\right)$ with reduction of singularities $\pi:(M, E) \rightarrow\left(\mathbb{C}^{2}, 0\right)$. The dual tree associated to $\mathcal{F}$ is the acyclic, double weighted, directed graph $\mathbb{A}^{*}(\mathcal{F})$ defined in the following way:

(1) to each component $D \subset E$ we associate a vertex $v(D)$;

(2) to $v(D)$ we associate weights $n_{1}(D) \in \mathbb{Z}_{<0}$ and $n_{2}(D) \in \mathbb{N} \cup\{\infty\}$, where $n_{1}(D)=$ $D \cdot D$ is the self-intersection number of $D$ in $M$ and $n_{2}(D)=\# \operatorname{Sep}(D)$;

(3) there is an arrow from $v\left(D_{2}\right)$ to $v\left(D_{1}\right)$ if and only if $D_{2} \cap D_{1} \neq \emptyset$ and $D_{2}$ results from a blow-up at a point in $D_{1}$.

The valence of a component $D \subset E$ is the number $\operatorname{Val}(D)$ of arrows of $\mathbb{A}^{*}(\mathcal{F})$ touching $v(D)$. In other words, it is the total number of components of $E$ intersecting $D$ other from $D$ itself.

Definition 2.3. Two foliations $\mathcal{F}$ and $\mathcal{F}^{\prime}$ are said to be equisingular or equireducible if $\mathbb{A}^{*}(\mathcal{F})=\mathbb{A}^{*}\left(\mathcal{F}^{\prime}\right)$

Let $\mathcal{F}$ be a foliation at $\left(\mathbb{C}^{2}, 0\right)$. A sequence of blow-ups $\pi:(M, E) \rightarrow\left(\mathbb{C}^{2}, 0\right)$ desingularizes $\operatorname{Sep}(\mathcal{F})$ if the transforms $\pi^{*} S$ of branches $S \in \operatorname{Sep}(\mathcal{F})$ are all disjoint and transverse to $E$. We call this map, which is supposed to be minimal, an $\mathcal{S}$-desingularization or $\mathcal{S}$-reduction for $\mathcal{F}$. Following the same procedure as in the construction of $\mathbb{A}^{*}(\mathcal{F})$, we define the $\mathcal{S}$-dual tree of $\mathcal{F}$, denoted as $\mathbb{A}_{\mathcal{S}}^{*}(\mathcal{F})$, as the dual tree associated to the $\mathcal{S}$-desingularization of $\mathcal{F}$. With this at hand, we have the following definitions:

Definition 2.4. A germ of foliation $\mathcal{F}$ is $\mathcal{S}$-desingularizable or $\mathcal{S}$-reducible if $\mathbb{A}_{\mathcal{S}}^{*}(\mathcal{F})=$ $\mathbb{A}^{*}(\mathcal{F})$, that is, an $\mathcal{S}$-desingularization actually is a desingularization for $\mathcal{F}$. 
Definition 2.5. Two germs of foliations $\mathcal{F}$ and $\mathcal{F}^{\prime}$ at $\left(\mathbb{C}^{2}, 0\right)$ are $\mathcal{S}$-equisingular or $\mathcal{S}$ equireducible if $\mathbb{A}_{\mathcal{S}}^{*}(\mathcal{F})=\mathbb{A}_{\mathcal{S}}^{*}\left(\mathcal{F}^{\prime}\right)$, that is, if their sets of separatrices have equivalent desingularizations.

\section{SECOND TYPE FOLIATIONS}

We keep the notation $\pi:(M, E) \rightarrow\left(\mathbb{C}^{2}, 0\right)$ for the reduction of singularities of $\mathcal{F}$ and $\tilde{\mathcal{F}}=\pi^{*} \mathcal{F}$ for the strict transform foliation. We say that a saddle-node singularity for $\tilde{\mathcal{F}}$ is tangent if its weak separatrix is contained in $E$. Non-tangent saddle-nodes are also known as well-oriented. The following definition is due to J.-F. Mattei and E. Salem (see [6] and

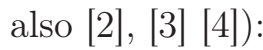

Definition 3.1. A foliation $\mathcal{F}$ at $\left(\mathbb{C}^{2}, 0\right)$ is of second type if there are no tangent saddlenodes in its reduction of singularities.

The main property of second type foliations to be used in this article is the following result, which already appeared in [6, Th. 3.1.9] in the non-dicritical case:

Proposition 3.2. Second type foliations are $\mathcal{S}$-desingularizable.

Proof. We first remark that the same proof of Lemma 1 in [1] applies to the following more general statement: a second type foliation with exactly two smooth transversal formal separatrices is simple. The result then follows by the same arguments as in the proof of [1, Th. 2].

We establish the following definition:

Definition 3.3. Two germs of foliations $\mathcal{F}$ and $\mathcal{F}^{\prime}$ at $\left(\mathbb{C}^{2}, 0\right)$ are topologically (respectively, $C^{\infty}$ ) equivalent if there is a germ of homeomorphism (respectively, $C^{\infty}$ diffeomorphism) $\Phi:\left(\mathbb{C}^{2}, 0\right) \rightarrow\left(\mathbb{C}^{2}, 0\right)$ which sends leaves of $\mathcal{F}$ on leaves of $\mathcal{F}^{\prime}$.

The family of second type foliations contains the subclass of generalized curve foliations, characterized by the absence saddle-nodes in the desingularization. The property of being a generalized curve foliation is a topological invariant and topological equivalent generalized curves are equisingular. This is the main result in |1|. Indeed, the topology of a generalized curve foliation is closely related to its separatrix set, entirely formed by convergent curves. The aim in Theorem [1] is to prove the equisingularity property for the family of second type foliations. If all separatrices of two second type foliations are convergent, then their topological equivalence implies equisingularity. Actually, there is a correspondence between homeomorphic separatrices for both foliations and the result follows from Zariski's equisingularity for curves in conjunction with the fact that a second type foliation is $\mathcal{S}$-desingularizable. However, in principle, a merely continuous equivalence map does not track purely formal separatrices. For this reason, in the statement of Theorem II] the regularity hypothesis on the equivalence map is strengthened and we ask for $C^{\infty}$ equivalences.

The following object was defined in [3]. A more thorough study on its properties is found in $\mid \mathbb{|}$. Again, $\mathcal{F}$ is a germ of foliation at $\left(\mathbb{C}^{2}, 0\right)$ with reduction process $\pi:(M, E) \rightarrow\left(\mathbb{C}^{2}, 0\right)$. 
Definition 3.4. A balanced equation of separatrices for $\mathcal{F}$ is a formal meromorphic function $\hat{F}$ whose associated divisor is

$$
(\hat{F})_{0}-(\hat{F})_{\infty}=\sum_{S \in \operatorname{Iso}(\mathcal{F})}(S)+\sum_{S \in \operatorname{Dic}(\mathcal{F})} a_{S}(S),
$$

where the coefficients $a_{S} \in \mathbb{Z}$ are non-zero only for finitely many $S \in \operatorname{Dic}(\mathcal{F})$, and, for each dicritical component $D \subset E$, the following equality holds:

$$
\sum_{S \in \operatorname{Sep}(\mathrm{D})} a_{S}=2-\operatorname{Val}(D)
$$

Note that if $\mathcal{F}$ is non-dicritical, then a balanced equation is an equation for the set of separatrices.

We recall that the multiplicity $\rho(D)$ of a component $D \subset E$ is defined as the algebraic multiplicity of a curve $\gamma$ at $\left(\mathbb{C}^{2}, 0\right)$ such that $\pi^{*} \gamma$ is transversal to $D$ outside a corner of $E$. We have the following definition:

Definition 3.5. The tangency excess of $\mathcal{F}$ along $E$ is the number

$$
\tau_{0}(\mathcal{F})=\sum_{q \in S N(\mathcal{F})} \rho\left(D_{q}\right)\left(\operatorname{Ind}_{q}^{w}(\tilde{\mathcal{F}})-1\right)
$$

where $S N(\mathcal{F}) \subset E$ denotes the set of all tangent saddle-nodes, $D_{q}$ is the component of $E$ containing the weak separatrix of $\tilde{\mathcal{F}}$ at $q \in S N(\mathcal{F})$ and $\operatorname{Ind}_{q}^{w}(\tilde{\mathcal{F}})>1$ is the weak index.

Note that $\tau_{0}(\mathcal{F}) \geq 0$ and, by definition, $\tau_{0}(\mathcal{F})=0$ if and only if $\operatorname{SN}(\mathcal{F})=\emptyset$, that is, if and only if $\mathcal{F}$ is of second type.

The algebraic multiplicity of a foliation $\mathcal{F}$ having $\omega=P d x+Q d y=0$ as a reduced equation is the integer $\nu_{0}(\mathcal{F})=\min \left(\nu_{0}(P), \nu_{0}(Q)\right)$. The tangency excess measures the extent that a balanced equation of separatrices computes the algebraic multiplicity of a foliation. This is expressed in the following fact, whose proof is found in [3]:

Proposition 3.6. Let $\mathcal{F}$ be a foliation on $\left(\mathbb{C}^{2}, 0\right)$ with $\hat{F}$ as a balanced equation of separatrices. Denote by $\nu_{0}(\mathcal{F})$ and $\nu_{0}(\hat{F})$ their algebraic multiplicities. Then

$$
\nu_{0}(\mathcal{F})=\nu_{0}(\hat{F})-1+\tau_{0}(\mathcal{F}) .
$$

We have, as a consequence:

Corollary 3.7. With the above notation,

$$
\nu_{0}(\mathcal{F})=\nu_{0}(\hat{F})-1
$$

if and only if $\mathcal{F}$ is a second type foliation.

\section{Pseudo-Analytic Curves}

Definition 4.1. Consider $\gamma:[0, \epsilon) \rightarrow \mathbb{R}^{k}(k \in \mathbb{N})$ with $\gamma(0)=0$. We say that the series

$$
\hat{\gamma}=\sum_{j=1}^{\infty} a_{j} t^{j}\left(a_{j} \in \mathbb{R}^{k}\right)
$$


is the Taylor series of $\gamma$ at $0 \in \mathbb{R}$ if, for each $n \in \mathbb{N}$, there is a function $\gamma_{n}:[0, \epsilon) \rightarrow \mathbb{R}^{k}$ with $\left|\gamma_{n}(t)\right|=o\left(t^{n}\right)$ and such that

$$
\gamma(t)=\sum_{j=1}^{n} a_{j} t^{j}+\gamma_{n}(t) .
$$

We say that $\hat{\gamma}$ is non-degenerate if $a_{j} \neq 0$ for some $j \in \mathbb{N}$.

Proposition 4.2. Suppose that $\gamma:[0, \epsilon) \rightarrow \mathbb{R}^{k}$ has a non-degenerate Taylor series $\hat{\gamma}$ at $0 \in \mathbb{R}$. Let $U$ and $U^{\prime}$ be neighborhoods of $0 \in \mathbb{R}^{k}$ such that $U$ contains the image of $\gamma$. Let $\Phi: U \rightarrow U^{\prime}$ be a $C^{\infty}$ diffeomorphism with $\Phi(0)=0$. Then the curve $\Phi \circ \gamma$ has a non-degenerate Taylor series at $0 \in \mathbb{R}$ which is given by the formal composition $\hat{\Phi} \circ \hat{\gamma}$, where $\hat{\Phi}$ is the Taylor series of $\Phi$ at $0 \in \mathbb{R}^{k}$.

This proposition, whose proof is left to the reader, allows us to establish the following definition:

Definition 4.3. Let $M$ be a $C^{\infty}$ manifold of dimension $k \in \mathbb{N}$ and consider the $C^{\infty}$ curve $\gamma:[0, \epsilon) \rightarrow M$ with $\gamma(0)=p \in M$. We say that $\gamma$ is pseudo-analytic at $p \in M$ if, for some $C^{\infty}$ chart $\psi$ with $\psi(p)=0 \in \mathbb{R}^{k}$, the curve $\psi \circ \gamma$ has a non-degenerate Taylor series at $0 \in \mathbb{R}$.

As a direct consequence of Proposition 4.2 we have:

Proposition 4.4. Let $M$ and $M^{\prime}$ be $C^{\infty}$ manifolds of dimension $k \in \mathbb{N}$ and let $\Phi: M \rightarrow$ $M^{\prime}$ be a $C^{\infty}$ diffeomorphism with $\Phi(p)=p^{\prime}$. Suppose that $\gamma:[0, \epsilon) \rightarrow M$ is pseudo-analytic at $p=\gamma(0)$. Then $\Phi \circ \gamma$ is pseudo-analytic at $p^{\prime} \in M^{\prime}$.

Next we show that the pseudo-analytic property is invariant under real blow-ups.

Proposition 4.5. Suppose that $\gamma:[0, \epsilon) \rightarrow M$ is injective and pseudo-analytic at $p=\gamma(0)$. Let $\pi: \tilde{M} \rightarrow M$ be the punctual real blow-up at $p \in M$. Then there exists $\tilde{p} \in \pi^{-1}(p)$ such the curve $\tilde{\gamma}=\pi^{-1} \circ \gamma:(0, \epsilon) \rightarrow \tilde{M}$ can be continuously extended by defining $\tilde{\gamma}(0)=\tilde{p}$. Moreover, the extended curve $\tilde{\gamma}:[0, \epsilon) \rightarrow \tilde{M}$ is injective and pseudo-analytic at $\tilde{p}$. Clearly, this proposition holds if $\pi$ is any finite composition of real blow-ups at $p \in M$.

Proof. Take $C^{\infty}$ coordinates $\left(x_{1}, \ldots, x_{k}\right)$ at $p \in M$. Then $\gamma=\left(\gamma_{1}, \ldots, \gamma_{k}\right)$ has a Taylor series

$$
\left(\sum_{j=\nu}^{\infty} a_{1}^{j} t^{j}, \ldots, \sum_{j=\nu}^{\infty} a_{k}^{j} t^{j}\right)
$$

with $\left(a_{1}^{\nu}, \ldots, a_{k}^{\nu}\right) \neq 0$. Of course we can assume that $a_{1}^{\nu} \neq 0$. If $a_{2}^{\nu} \neq 0$, define the diffeomorphism

and consider

$$
\psi:\left(x_{1}, \ldots, x_{k}\right) \mapsto\left(x_{1}, x_{2}-\frac{a_{2}^{\nu}}{a_{1}^{\nu}} x_{1}, x_{3}, \ldots, x_{k}\right)
$$

$$
\psi \circ \gamma(t)=\left(\tilde{\gamma_{1}}(t), \tilde{\gamma_{2}}(t), \ldots, \tilde{\gamma}_{k}(t)\right)
$$

Then it is easy to see that $\operatorname{ord}\left(\hat{\tilde{\gamma_{2}}}\right)>\nu$, where $\hat{\tilde{\gamma_{2}}}$ is the Taylor series of $\tilde{\gamma_{2}}$. Therefore, by changing coordinates if necessary we can assume that $a_{1}^{\nu} \neq 0$ and $a_{j}^{\nu}=0$ for $j=2, \ldots, k$. 
Thus, for $t>0$ small we have

$$
\pi^{-1} \circ \gamma(t)=\left(\gamma_{1}(t), \frac{\gamma_{2}(t)}{\gamma_{1}(t)}, \ldots, \frac{\gamma_{k}(t)}{\gamma_{1}(t)}\right)
$$

which clearly tends to $(0, \ldots, 0)$ as $t \rightarrow 0$. Since $\gamma_{1}$ has a non-degenerate Taylor series, it suffices to show that $\frac{\gamma_{j}(t)}{\gamma_{1}(t)}$ has a Taylor series for $j=2, \ldots, k$. We will show that the formal quotient $\sum_{j=0}^{\infty} q_{j} t^{j}$ of $\sum_{j=\nu}^{\infty} a_{2}^{j} t^{j}$ by $\sum_{j=\nu}^{\infty} a_{1}^{j} t^{j}$ is the Taylor series of $\frac{\gamma_{2}}{\gamma_{1}}$ at $t=0$; the other cases are equal. Fix $n \in \mathbb{N}$. It is sufficient to show that

$$
R:=\frac{\gamma_{2}(t)}{\gamma_{1}(t)}-\sum_{j=0}^{n} q_{j} t^{j}=o\left(t^{n}\right)
$$

We can express

$$
\begin{aligned}
& \gamma_{1}(t)=\sum_{j=\nu}^{\nu+n} a_{1}^{j} t^{j}+f_{1}(t), \\
& \gamma_{2}(t)=\sum_{j=\nu}^{\nu+n} a_{2}^{j} t^{j}+f_{2}(t),
\end{aligned}
$$

where $f_{1}(t), f_{2}(t)=o\left(t^{\nu+n}\right)$. Then

$$
\begin{aligned}
\frac{R}{t^{n}} & =\frac{\gamma_{2}(t)-\gamma_{1}(t) \sum_{j=0}^{n} q_{j} t^{j}}{t^{n} \gamma_{1}(t)} \\
& =\frac{\left(\sum_{j=\nu}^{\nu+n} a_{2}^{j} t^{j}+f_{2}(t)\right)-\left(\sum_{j=\nu}^{\nu+n} a_{1}^{j} t^{j}+f_{1}(t)\right) \sum_{j=0}^{n} q_{j} t^{j}}{t^{n} \gamma_{1}(t)} \\
& =\frac{o\left(t^{\nu+n}\right)}{t^{n} \gamma_{1}(t)}=\frac{o\left(t^{\nu+n}\right)}{t^{\nu+n}} \frac{1}{\gamma_{1}(t) / t^{\nu}} \rightarrow 0 \text { as } t \rightarrow 0 .
\end{aligned}
$$

\section{Pseudo-Analytic CURVES in COMPlex Surfaces}

Let $V$ be a complex regular surface and consider a curve $\gamma:[0, \epsilon) \rightarrow V$ pseudo-analytic at $p=\gamma(0) \in V$. In local holomorphic coordinates at $p$ the Taylor series of $\gamma$ is given as

$$
\hat{\gamma}=\left(\sum_{j=1}^{\infty} a_{j} t^{j}+i \sum_{j=1}^{\infty} b_{j} t^{j}, \sum_{j=1}^{\infty} c_{j} t^{j}+i \sum_{j=1}^{\infty} d_{j} t^{j}\right),
$$

where $a_{j}, b_{j}, c_{j}, d_{j} \in \mathbb{R}$. Then, if we set $\alpha_{j}=a_{j}+i b_{j}$ and $\beta_{j}=c_{j}+i d_{j}$, we can write

$$
\hat{\gamma}=\left(\sum_{j=1}^{\infty} \alpha_{j} t^{j}, \sum_{j=1}^{\infty} \beta_{j} t^{j}\right)
$$


Thus, converting the real variable $t \in \mathbb{R}$ into a complex variable $z \in \mathbb{C}$, we can view the Taylor series of $\gamma$ as the formal complex parametrized curve

$$
\left(\sum_{j=1}^{\infty} \alpha_{j} z^{j}, \sum_{j=1}^{\infty} \beta_{j} z^{j}\right)
$$

at $p \in V$.

Definition 5.1. Let $\gamma$ be a pseudo-analytic curve at $p \in V$ and let $\mathcal{C}$ be a formal parametrized complex curve at $p \in V$. We say that $\gamma$ is asymptotic to $\mathcal{C}$ at $p \in V$ if $\hat{\gamma}$ is a formal reparametrization of $\mathcal{C}$, that is, if there exists a formal invertible complex series $\hat{\psi}=\sum_{j=1}^{\infty} \sigma_{j} w^{j}$ such that $\hat{\gamma}=\mathcal{C} \circ \hat{\psi}$.

Proposition 5.2. Let $\gamma$ be an injective pseudo-analytic curve at $p \in V$, which is asymptotic to a formal complex curve $\mathcal{C}$ at $p \in V$. Let $\pi: \tilde{V} \rightarrow V$ be the punctual complex blow-up at $p \in V$. Then there exists $\tilde{p} \in \pi^{-1}(p)$ such the curve $\tilde{\gamma}=\pi^{-1} \circ \gamma:(0, \epsilon) \rightarrow \tilde{V}$ can be continuously extended by defining $\tilde{\gamma}(0)=\tilde{p}$. Moreover:

(1) the extended curve $\tilde{\gamma}:[0, \epsilon) \rightarrow \tilde{V}$ is injective and pseudo-analytic at $\tilde{p}$;

(2) $\tilde{\gamma}$ is asymptotic at $\tilde{p}$ to the strict transform of $\mathcal{C}$ by $\pi$.

Clearly, this proposition holds if $\pi$ is any finite composition of complex blow-ups at $p \in V$.

Proof. Take holomorphic coordinates $\left(z_{1}, z_{2}\right)$ at $p \in V$. Then $\gamma=\left(\gamma_{1}, \gamma_{2}\right)$ has a Taylor series

$$
\left(\sum_{j=\nu}^{\infty} a_{1}^{j} t^{j}, \sum_{j=\nu}^{\infty} a_{2}^{j} t^{j}\right)
$$

with $a_{1}^{j}, a_{2}^{j} \in \mathbb{C}$ and $\left(a_{1}^{\nu}, a_{2}^{\nu}\right) \neq 0$. Of course we can assume that $a_{1}^{\nu} \neq 0$. Moreover, as in the proof of Proposition 4.5, by changing coordinates if necessary we can assume that $a_{2}^{\nu}=0$. Thus, for $t>0$ small we have

$$
\pi^{-1} \circ \gamma(t)=\left(\gamma_{1}(t), \frac{\gamma_{2}(t)}{\gamma_{1}(t)}\right)
$$

which clearly tends to $(0,0)$ as $t \rightarrow 0$. Since $\gamma_{1}$ has a non-degenerate Taylor series, in order to prove item (11) it suffices to show that $\frac{\gamma_{2}(t)}{\gamma_{1}(t)}$ has a Taylor series at $t=0$. In fact, proceeding as in Proposition 4.5, we show that the Taylor series of $\frac{\gamma_{2}(t)}{\gamma_{1}(t)}$ is given by the formal quotient of $\sum_{j=\nu}^{\infty} a_{2}^{j} t^{j}$ by $\sum_{j=\nu}^{\infty} a_{1}^{j} t^{j}$ and this also implies item (2).

Definition 5.3. Let $\mathcal{F}$ be a one-dimensional holomorphic foliation on a complex regular surface $V$, with a singularity at $p \in V$. Consider a $C^{\infty}$ curve $\gamma:[0, \epsilon) \rightarrow V$ with $\gamma(0)=0$. We say that $\gamma$ is a characteristic curve of $\mathcal{F}$ at $p$ if the following properties hold:

(1) $\gamma$ is injective and pseudo-analytic at $p \in V$;

(2) $\gamma((0, \epsilon))$ is contained in a leaf of $\mathcal{F}$.

A characteristic curve of a foliation is canonically asymptotic to a separatrix, as shown in the following result: 
Proposition 5.4. Let $\mathcal{F}$ be a one-dimensional holomorphic foliation on a complex regular surface $V$, with a singularity at $p \in V$. If $\gamma:[0, \epsilon) \rightarrow V$ is a characteristic curve of $\mathcal{F}$ at $p$, then $\hat{\gamma}$ is a parametrized formal separatrix of $\mathcal{F}$.

Proof. Let $\omega$ be a holomorphic one form defining $\mathcal{F}$ near $p \in V$. Since $\gamma(t)$ is contained in a leaf of $\mathcal{F}$ for $t \in(0, \epsilon)$, we have that

$$
\omega(\gamma(t)) \cdot \gamma^{\prime}(t)=0 \text { for all } t \in[0, \epsilon) .
$$

Then the Taylor series of $\omega(\gamma(t)) \cdot \gamma^{\prime}(t)$ at $t=0$ is null, that is,

$$
\hat{\omega}(\hat{\gamma}) \cdot \hat{\gamma}^{\prime}=0
$$

We finish this section providing, in three examples, an analysis of characteristic curves according to the type of separatrices to which they are asymptotic.

Example 5.5. Characteristic curves asymptotic to a dicritical separatrix. Let $\gamma$ be a characteristic curve asymptotic to a dicritical separatrix $S$ of a foliation $\mathcal{F}$. After the desingularization of $\mathcal{F}$, the strict transforms of $S$ and $\gamma$ tend to a trace point in a dicritical component. Thus, the only possibility is that $\gamma$ is a curve contained in $S$.

Example 5.6. Characteristic curves asymptotic to a strong separatrix. Let $\gamma$ be a characteristic curve asymptotic to a strong separatrix $S$ of a foliation $\mathcal{F}$. Let $\pi:(M, E) \rightarrow\left(\mathbb{C}^{2}, 0\right)$ be the desingularization of $\mathcal{F}$ and denote by $\tilde{\mathcal{F}}=\pi^{*} \mathcal{F}$ the strict transform of $\mathcal{F}$. Then the strict transform $\tilde{S}=\pi^{*} S$ is a separatrix of some reduced singularity $p \in E$ of $\tilde{\mathcal{F}}$. Since the separatrix $S$ is strong, by doing one more blow-up at $p$ if it were a saddle node, we can assume that the singularity at $p$ is non degenerate. Moreover, by performing some additional blow-ups if necessary, we can assume that the ratio of eigenvalues of the singularity at $p$ has a negative real part. Thus, we can take holomorphic local coordinates $(u, v)$ at $p$ such that:

(1) the foliation $\tilde{\mathcal{F}}$ at $p$ is generated by a 1 -form

$$
\omega=u d v-v Q(u, v) d u,
$$

where $Q(u, v)=\lambda+\ldots, \operatorname{Re}(\lambda)<0$.

(2) $E$ is given by $\{u=0\}$;

(3) $\tilde{S}$ is given by $\{v=0\}$.

Let $\tilde{\gamma}$ be the strict transform of $\gamma$ by $\pi$. By Proposition 5.2, $\tilde{\gamma}$ is a characteristic curve of $\tilde{\mathcal{F}}$ asymptotic to $\tilde{S}$. We will prove that $\tilde{\gamma}$ is contained in $S$. If we express $\tilde{\gamma}(t)=(u(t), v(t))$, $t \in[0, \epsilon)$, since $\tilde{\gamma}$ is tangent to the foliation $\tilde{\mathcal{F}}$, we have that

$$
u(t) v^{\prime}(t)-v(t) Q(u(t), v(t)) u^{\prime}(t)=0 .
$$

Then, if we define $r(t)=|v(t)|^{2}$, a straightforward computation give us that

$$
r^{\prime}=2|v|^{2} \operatorname{Re}\left(\frac{u^{\prime}}{u} Q\right)
$$

Since $\tilde{\gamma}$ has a nonzero Taylor series and $\tilde{\gamma}$ is asymptotic to $\{v=0\}$, we see that $u(t)$ has a nonzero Taylor series $\hat{u}(t)=\sum_{j \geq n} a_{j} t^{j}, a_{j} \in \mathbb{C}, a_{n} \neq 0, n \in \mathbb{N}$. From this we easily obtain 
that

and therefore

$$
\frac{u^{\prime}(t)}{u(t)}=\frac{1}{t}(n+o(t))
$$

$$
r^{\prime}=2|v|^{2} \frac{1}{t} \operatorname{Re}((n+o(t)) Q)
$$

for all $t \in(0, \epsilon)$. Suppose that $\tilde{\gamma}$ is not contained in $\tilde{S}$. Then we have $|v(t)|>0$ for all $t \in(0, \epsilon)$. Thus, since

$$
\operatorname{Re}((n+o(t)) Q) \rightarrow n \operatorname{Re}(\lambda)<0,
$$

for $t>0$ small enough we have that $r^{\prime}(t)<0$. But this is a contradiction, since that $r(0)=0$ and $r(t)>0$ for $t \in(0, \epsilon)$. Therefore, we conclude that a strong separatrix contains all its asymptotic characteristic curves.

Example 5.7. Characteristic curves asymptotic to a weak separatrix. Consider a saddlenode foliation $\mathcal{F}$ at $0 \in \mathbb{C}^{2}$ whose strong separatrix is contained in $\{(u, v): u=0\}$. Then there is a formal series $\hat{s}(u)=\sum_{j=1}^{\infty} c_{j} u^{j}$ such that the weak separatrix $S$ of $\mathcal{F}$ is given by $v=\hat{s}(u)$. It is known that there exists a constant $\vartheta>0$ depending only on the analytic type of the saddle-node such that, given $\eta \in \mathbb{C}^{*}$, we can find a holomorphic function $f$ defined on a sector of the form

$$
V=\left\{r e^{i \theta} \eta \in \mathbb{C}: 0<r<\epsilon,-\vartheta<\theta<\vartheta\right\}(\epsilon>0)
$$

such that:

(1) the graph $\{(u, f(u)): u \in V\}$ is contained in a leaf of the foliation $\mathcal{F}$;

(2) the function $f$ has the series $\sum_{j=1}^{\infty} c_{j} u^{j}$ as asymptotic expansion at $0 \in \mathbb{C}$.

Define

$$
\gamma(t)=(\eta t, f(\eta t))
$$

for $t \geq 0$ small and observe that the Taylor series of $\gamma$ at $t=0$ is given by $(\eta t, \hat{s}(\eta t))$. Therefore $\gamma$ is a characteristic curve of $\mathcal{F}$ asymptotic to the separatrix $S$. We remark that the function $f$ above can be non unique, for example if $\eta$ corresponds to a "node" direction of the saddle-node. Thus, even if the weak separatrix is convergent, it does not contain all its asymptotic characteristic curves. In general, the argument above can be done for a saddle-node appearing in the desingularization of a non reduced foliation $\mathcal{F}$. Thus, we conclude that a weak separatrix $S$ of any foliation does not contain all its asymptotic characteristic curves, even if $S$ is convergent.

\section{Correspondence of Separatrices by a $C^{\infty}$ equivalence}

The main result of this section is the following:

Theorem 6.1. Let $\Phi$ be a $C^{\infty}$ equivalence between two germs $\mathcal{F}$ and $\mathcal{F}^{\prime}$ of singular holomorphic foliations at $\left(\mathbb{C}^{2}, 0\right)$. Then, given a formal separatrix $S$ of $\mathcal{F}$, there exists a unique formal separatrix $S^{\prime}$ of $\mathcal{F}^{\prime}$, denoted by $S^{\prime}=\Phi_{*}(S)$, such that for any characteristic curve $\gamma$ of $\mathcal{F}$ asymptotic to $S$ we have that $\gamma^{\prime}=\Phi(\gamma)$ is a characteristic curve of $\mathcal{F}^{\prime}$ asymptotic to $S^{\prime}$.

In order to prove the theorem, we need two preliminary results. 
Lemma 6.2. Fix $\nu \in \mathbb{N}$. Then, for each $j \in \mathbb{N}$ there exists a complex polynomial $P_{j}$ in $2 j+1$ variables such that, if

$$
\hat{S}=\left(\sum_{j=\nu}^{\infty} a_{j} z^{j}, \sum_{j=\nu}^{\infty} b_{j} z^{j}\right)
$$

is a parametrized formal complex curve with $a_{\nu}=c^{\nu}, c \neq 0$, and we set

$$
\sigma_{j}=P_{j}\left(c, \frac{1}{c}, a_{\nu+1}, \ldots, a_{\nu+j-1}, b_{\nu}, \ldots, b_{\nu+j-1}\right),
$$

then $S$ can be reparametrized as

$$
\left(x^{\nu}, \sum_{j=\nu}^{\infty} \sigma_{j} x^{j}\right),
$$

that is, there is an invertible series $\hat{\psi}(x)$ such that $\hat{S}(\psi(x))=\left(x^{\nu}, \sum_{j=\nu}^{\infty} \sigma_{j} x^{j}\right)$.

Proof. This lemma is only a Puiseux's Parametrization putting in evidence the dependence of the final coefficients in terms of the initial ones.

Proposition 6.3. Let $\Phi$ be a $C^{\infty}$ equivalence between two holomorphic foliations with isolated singularity at $0 \in \mathbb{C}^{2}$ and let $J: \mathbb{C}^{2} \rightarrow \mathbb{C}^{2}$ be the complex conjugation. Then either $d \Phi(0)$ or $d \Phi(0) \circ J$ is a $\mathbb{C}$-linear isomorphism of $\mathbb{C}^{2}$.

Proof. See [9, Lemma 4.3].

Proof of Theorem 6.1. If $\gamma$ is a characteristic curve of $\mathcal{F}$ asymptotic to $S$, it is obvious that $\Phi(\gamma)$ is a characteristic curve of $\mathcal{F}^{\prime}$. Moreover, by Proposition 5.4 the characteristic curve $\Phi(\gamma)$ is asymptotic to the formal separatrix given by its Taylor series. The nontrivial fact is that this formal separatrix depends only on $S$ and not on the characteristic curve $\gamma$.

If $S$ is convergent we take $S^{\prime}=\Phi(S)$. The proof of the theorem will then be easy in the following cases:

(1) $S$ is a dicritical separatrix;

(2) $S$ is a strong separatrix.

In both cases, if $\gamma$ is a characteristic curve asymptotic to $S$, by examples 5.5 and 5.6 we have that $\gamma \subset S$. Since $\Phi(\gamma)$ is contained in $S^{\prime}$, then $\Phi(\gamma)$ is a characteristic curve asymptotic to $S^{\prime}$.

We begin the proof of the remaining case. Let $\pi:(M, E) \rightarrow\left(\mathbb{C}^{2}, 0\right)$ be the reduction of singularities of $\mathcal{F}$. Then, the strict transform $\tilde{S}=\pi^{*} S$ is the weak separatrix of a saddle-node singularity at some trace point $p \in E$. Clearly $p$ is not a corner and its strong separatrix is contained in $E$. Let $(u, v)$ be local holomorphic coordinates at $p \in E$ such that:

(1) $p \simeq(0,0)$;

(2) $E$ is given by $\{u=0\}$.

Then there exists a formal series $\hat{s}(u)=\sum_{j=1}^{\infty} c_{j} u^{j}$ such that the $\tilde{S}$ is given by $v=\hat{s}(u)$. Let $\gamma$ be a characteristic curve asymptotic to $S$ and let $\tilde{\gamma}(t)=(u(t), v(t))$ be the strict 
transform of $\gamma$ by $\pi$. Then $\tilde{\gamma}$ has a non-degenerate Taylor series given by

$$
(\hat{u}, \hat{v})=\left(\sum_{j=1}^{\infty} u_{j} t^{j}, \sum_{j=1}^{\infty} v_{j} t^{j}\right), \text { where } u_{j}, v_{j} \in \mathbb{C} .
$$

Since $\tilde{\gamma}$ is asymptotic to $\tilde{S}$ at $p$, we deduce that $u_{1} \neq 0$ and $\hat{v}=\hat{s} \circ \hat{u}$. Let $\hat{\pi}=\hat{\pi}_{\nu}+\hat{\pi}_{\nu+1}+\ldots$ be the Taylor series of $\pi$ at $p$, where the $\hat{\pi}_{\nu}$ is the initial part of $\pi$. It is easy to see that the initial part of $\hat{\tilde{\gamma}}=(\hat{u}, \hat{s} \circ \hat{u})$ is given by $\hat{\tilde{\gamma}}_{1}=\left(u_{1} t, c_{1} u_{1} t\right)$. Then the initial part of $\hat{\gamma}=\hat{\pi} \circ \hat{\tilde{\gamma}}$ is $\hat{\pi}_{\nu}\left(u_{1}, c_{1} u_{1}\right) t^{\nu}$. Since $\Phi$ is a diffeomorphism, its initial part $\hat{\Phi}_{1}$ is an isomorphism, so

$$
\hat{\Phi}_{1} \circ \hat{\pi}_{\nu}\left(u_{1}, c_{1} u_{1}\right) \neq 0 .
$$

Then the initial part of $\hat{\gamma}^{\prime}=\hat{\Phi} \circ \hat{\gamma}$ is

$$
\hat{\gamma}_{\nu}^{\prime}=\hat{\Phi}_{1} \circ \hat{\pi}_{\nu}\left(u_{1}, c_{1} u_{1}\right) t^{\nu} .
$$

Thus, we can write

$$
\hat{\gamma}^{\prime}=\left(\sum_{j=\nu}^{\infty} a_{j} t^{j}, \sum_{j=\nu}^{\infty} b_{j} t^{j}\right)
$$

where the coefficients $a_{j}$ and $b_{j}$ are polynomials in the coefficients of $\operatorname{Re}(\hat{u})$ and $\operatorname{Im}(\hat{u})$ and $\left(a_{\nu}, b_{\nu}\right) \neq 0$. If $J: \mathbb{C}^{2} \rightarrow \mathbb{C}^{2}$ is the complex conjugation, by Proposition 6.3 we have that either $\Phi_{1}$ or $\Phi_{1} \circ J$ is a $\mathbb{C}$-linear isomorphism. Then there is $(a, b) \in \mathbb{C}^{2} \backslash\{0\}$ such that

(1) $\left(a_{\nu}, b_{\nu}\right)=\left(a u_{1}^{\nu}, b u_{1}^{\nu}\right)$, or

(2) $\left(a_{\nu}, b_{\nu}\right)=\left(a \overline{u_{1}}{ }^{\nu}, b \overline{u_{1}}{ }^{\nu}\right)$.

Both cases are similar, so we only deal with the first one. Of course we can suppose that $a \neq 0$, so $a_{\nu} \neq 0$ for all $u_{1} \in \mathbb{C}^{*}$. Since $\gamma^{\prime}$ is a characteristic curve of $\mathcal{F}^{\prime}$, by Proposition 5.4 the formal curve

$$
\hat{\gamma^{\prime}}=\left(\sum_{j=\nu}^{\infty} a_{j} z^{j}, \sum_{j=\nu}^{\infty} b_{j} z^{j}\right)
$$

is a parametrization of a formal separatrix $S_{\hat{u}}^{\prime}$ of $\mathcal{F}^{\prime}$. Moreover, $S_{\hat{u}}^{\prime}$ is an isolated separatrix, otherwise $\gamma$ should be contained in a dicritical separatrix of $\mathcal{F}^{\prime}$. We can apply Lemma 6.2 in order to obtain a parametrization

$$
S_{\hat{u}}^{\prime}=\left(x^{\nu}, \sum_{j=\nu}^{\infty} \sigma_{j} x^{j}\right),
$$

where the coefficients $\sigma_{j}$ are polynomials in $1 / u_{1}$ and in the coefficients of re $(\hat{u})$ and $\operatorname{im}(\hat{u})$. Consider the map

$$
\phi: \hat{u} \mapsto\left(\sigma_{\nu}, \sigma_{\nu+1}, \ldots\right) .
$$

Clearly we can identify the set of formal complex series in one variable with $\mathbb{C}^{\mathbb{N}}$. Then the function $\phi$ is defined in some subset $\hat{U}$ of $\mathbb{C}^{*} \times \mathbb{C}^{\mathbb{N}}$ and take values in the set

$$
\Sigma=\left\{\left(\sigma_{\nu}, \sigma_{\nu+1}, \ldots\right) \in \mathbb{C}^{\mathbb{N}}:\left(x^{\nu}, \sum_{j=\nu}^{\infty} \sigma_{j} x^{j}\right) \in \operatorname{Iso}\left(\mathcal{F}^{\prime}\right)\right\},
$$

where $\operatorname{Iso}\left(\mathcal{F}^{\prime}\right)$ is the set of isolated separatrices of $\mathcal{F}^{\prime}$. Observe that the set $\Sigma$ is finite and $\phi$ is continuous if we consider the product topology in $\mathbb{C}^{\mathbb{N}}$. Then it is sufficient to prove that 
$\hat{U}$ is connected, because in this case the map $\phi$ is constant and we can define $S^{\prime}=S_{\hat{u}}^{\prime}$ for any $\hat{u} \in \hat{U}$. We will prove that $\hat{U}$ is path connected. From Example 5.7, for any $u_{1} \in \mathbb{C}^{*}$ there exists a characteristic curve of $\mathcal{F}$ whose strict transform by $\pi$ has a Taylor series at $p \in M$ given by $\left(u_{1} t, \hat{s}\left(u_{1} t\right)\right)$. This shows that $C^{*}:=\mathbb{C}^{*} \times\{0\}^{\mathbb{N}}$ is contained in $\hat{U}$. Since this set is path connected, it suffices to show that any $\hat{u} \in \hat{U}$ can be connected with some point in $C^{*}$ by a continuous path. Fix $\hat{u}=\left(u_{1}, \ldots\right) \in \hat{U}$. Then there exists a characteristic curve $\gamma$ of $\mathcal{F}$ such that its strict transform $\tilde{\gamma}=(u(t), v(t))$ by $\pi$ has $(\hat{u}, \hat{s} \circ \hat{u})$ as its Taylor series at $p \in M$. We can assume that the image of $u(t)$ is contained in a sector of the form

$$
V=\left\{r e^{i \theta} \in \mathbb{C}: 0<r<\epsilon, a<\theta<b\right\}(\epsilon, a, b>0)
$$

such that there exists a function $f \in \mathcal{O}(V)$ with the following properties:

(1) the graph $\{(u, f(u)): u \in V\}$ is contained in a leaf of the foliation;

(2) the function $f$ has the series $\hat{s}=\sum_{j=1}^{\infty} c_{j} u^{j}$ as asymptotic expansion at $0 \in \mathbb{C}$.

Consider $\tilde{\gamma_{0}}(t)=(u(t), f(u(t)))$ and $\tilde{\gamma}_{1}(t)=\left(u_{1} t, f\left(u_{1} t\right)\right)$ and observe the following:

(1) $\tilde{\gamma}_{0}$ and $\tilde{\gamma}_{1}$ are the strict transforms by $\pi$ of characteristic curves of $\mathcal{F}$ asymptotic to $S$;

(2) $\tilde{\gamma}_{0}$ has $(\hat{u}, \hat{s} \circ \hat{u})$ as its Taylor series;

(3) If $\hat{u}_{1}:=\left(u_{1}, 0, \ldots\right) \in C^{*}$, then $\tilde{\gamma}_{1}$ has $\left(\hat{u}_{1}, \hat{s} \circ \hat{u}_{1}\right)$ as its Taylor series.

Define the family of curves

$$
\Gamma_{s}(t)=\left((1-s) u(t)+s u_{1} t, f\left((1-s) u(t)+s u_{1} t\right)\right), s \in[0,1] .
$$

It is easy to see that

(1) $\Gamma_{0}=\tilde{\gamma_{0}}$ and $\Gamma_{1}=\tilde{\gamma_{1}}$

(2) each $\Gamma_{s}$ is the strict transform of a characteristic curve asymptotic to $S$;

(3) $\hat{\Gamma}_{s}=\left(\hat{u}_{s}, \hat{s} \circ \hat{u}_{s}\right)$, where $\hat{u}_{s}=u_{1}+\sum_{j=2}^{\infty}(1-s) u_{j} t^{j}$.

Then $\hat{u}_{s}$ defines a continuous path connecting $\hat{u}$ with $\hat{u}_{1} \in C^{*}$.

\section{Formal ReAl EQUivalence AND EQUisingularity FOR CURVES}

In this section we introduce the notion of formal real equivalence for formal complex curves at $\left(\mathbb{C}^{2}, 0\right)$ and we prove that this notion implies the equisingularity property (Theorem 7.2). A formal parametrized real surface at $\left(\mathbb{C}^{2}, 0\right)$ is a nonzero series in two variables of the form

$$
\sum_{j, k \in \mathbb{N}} a_{j k} x^{j} y^{k}
$$

where $a_{j k} \in \mathbb{C}^{2}$ for all $j, k \in \mathbb{N}$. Naturally, a formal parametrized complex curve $\sum_{j \in \mathbb{N}} \alpha_{j} z^{j}$ $\left(\alpha_{j} \in \mathbb{C}^{2}\right)$ is also a formal parametrized real surface if we do the substitution $z=x+i y$. A formal real reparametrization of the surface 7.1 is any series obtained by a substitution $(x, y)=\Psi(\bar{x}, \bar{y})$, where $\Psi$ is a formal diffeomorphism of $\left(\mathbb{R}^{2}, 0\right)$.

Definition 7.1. Let $\hat{\Phi}$ be a formal diffeomorphism of $\left(\mathbb{R}^{4}, 0\right)$. Let $\sigma(z)=\sum_{j \in \mathbb{N}} \sigma_{j} z^{j}$ and $\sigma^{\prime}(z)=\sum_{j \in \mathbb{N}} \sigma_{j}^{\prime} z^{j}$ be two formal parametrized irreducible complex curves at $\left(\mathbb{C}^{2}, 0\right)$. 
We say that $\hat{\Phi}$ is a formal real equivalence between $\sigma$ and $\sigma^{\prime}$ if $\hat{\Phi} \circ \sigma$ is a formal real reparametrization of $\sigma^{\prime}$. In this situation we also say that $\sigma$ and $\sigma^{\prime}$ are formally real equivalent by $\hat{\Phi}$. In general, we say that $\hat{\Phi}$ is a formal real equivalence between two reduced formal complex curves $\mathscr{C}$ and $\mathscr{C}^{\prime}$ at $\left(\mathbb{C}^{2}, 0\right)$ if there is bijection between the irreducible components of $\mathscr{C}$ with the irreducible components of $\mathscr{C}^{\prime}$ such that each pair of corresponding irreducible components are formally real equivalent by $\hat{\Phi}$.

Theorem 7.2. Let $\hat{\Phi}$ be a formal real equivalence between two germs of reduced formal complex curves $\mathscr{C}$ and $\mathscr{C}^{\prime}$ at $\left(\mathbb{C}^{2}, 0\right)$. Then $\mathscr{C}$ and $\mathscr{C}^{\prime}$ are equisingular.

Proof. Let $\xi^{1}, \ldots, \xi^{m}$ and $\xi^{\prime 1}, \ldots, \xi^{\prime m}$ be the irreducible components of $\mathscr{C}$ and $\mathscr{C}^{\prime}$ respectively and assume that $\hat{\Phi}$ maps $\xi^{k}$ to $\xi^{\prime k}$ for $k=1, \ldots, m$. Let

$$
\sigma_{k}(z)=\sum_{j \geq 1} a_{j}(k) z^{j}, a_{j}(k) \in \mathbb{C}^{2}
$$

be a formal parametrization of $\xi^{k}$. Then $\hat{\Phi} \circ \sigma_{k}$ is a real formal parametrization of the irreducible component $\xi^{\prime k}$ of $\mathscr{C}^{\prime}$, that is, there exists a real formal diffeomorphism $\psi_{k}:(\mathbb{C}, 0) \rightarrow(\mathbb{C}, 0)$ such that $\hat{\Phi} \circ \sigma_{k} \circ \psi_{k}(z)$ is a complex formal parametrization of $\xi^{\prime k}$. Given $n \in \mathbb{N}$, let $\xi_{n}^{k}$ and $\xi_{n}^{\prime k}$ be the complex curves defined by the $n$-jets of $\sigma_{k}(z)$ and $\hat{\Phi} \circ \sigma_{k} \circ \psi_{k}(z)$ respectively. Let $\mathscr{C}_{n}$ and $\mathscr{C}_{n}^{\prime}$ be the reduced curves whose irreducible components are $\left\{\xi_{n}^{k}: k=1, \ldots, m\right\}$ and $\left\{\xi_{n}^{\prime k}: k=1, \ldots, m\right\}$, respectively. We know that for $n$ large enough:

(1) $\mathscr{C}$ and $\mathscr{C}_{n}$ are equisingular;

(2) $\mathscr{C}^{\prime}$ and $\mathscr{C}_{n}^{\prime}$ are equisingular.

Then it is sufficient to prove that the analytic curves $\mathscr{C}_{n}$ and $\mathscr{C}_{n}^{\prime}$ are topologically equivalent for $n$ large enough. For the sake of simplicity we denote $\xi^{1}, \xi^{\prime 1}, \xi_{n}^{1}, \xi_{n}^{\prime 1}, \sigma_{1}$ and $\psi_{1}$ by $\xi$, $\xi^{\prime}, \xi_{n}, \xi_{n}^{\prime}, \sigma$ and $\psi$, respectively. Then $\hat{\Phi} \circ \sigma \circ \psi(z)$ is a complex formal parametrization of $\xi^{\prime}$ and $\xi_{n}$ is defined by the $n$-jet $\sigma_{n}$ of $\sigma$. Since the curves $\xi_{n}$ and $\xi_{n}^{\prime}$ are analytic, we will use the same notation for the sets defined by these curves. If $\Phi_{n}$ is the $n$-jet of $\Phi$, the singular real surface $\mathscr{S}$ given by $\Phi_{n}\left(\xi_{n}\right)$ is asymptotic to $\hat{\Phi} \circ \sigma \circ \psi(z)$ up to order $n$. In fact, if we consider the $n$-jet $\psi_{n}$ of $\psi$, the real parametrization $\Phi_{n} \circ \sigma_{n} \circ \psi_{n}(z)$ of $\Phi_{n}\left(\xi_{n}\right)$ has a Taylor series coinciding with the Taylor series of $\hat{\Phi} \circ \sigma \circ \psi(z)$ up to order $n$. After a finite sequence of complex blow-ups $\pi:(M, E) \rightarrow\left(\mathbb{C}^{2}, 0\right)$, the strict transform $\tilde{\xi}^{\prime}$ of $\xi^{\prime}$ is a regular formal curve transverse to the exceptional divisor $E$ at a point $p$. Let $(x, y)$ be holomorphic coordinates on a neighborhood of $p$ such that:

(1) $p \simeq(0,0)$;

(2) the exceptional divisor $E$ is given by $\{x=0\}$;

(3) the curve $\tilde{\xi}^{\prime}$ is given by a formal equation $y=\sum_{j \geq 1} c_{j} x^{j}$.

The following properties hold for $n$ large enough:

(1) the strict transform $\tilde{\xi_{n}^{\prime}}$ of $\xi_{n}^{\prime}$ by $\pi$ intersects $E$ at the point $p$ and is given by an analytic equation of the form $y=\zeta(x)=c_{1} x+o(x)$ near of $p$;

(2) the strict transform $\tilde{\mathscr{S}}$ of $\mathscr{S}$ by $\pi$ intersects $E$ at the point $p$ and is given by a $C^{\infty}$ equation of the form $y=f(x)=c_{1} x+o(x)$ near of $p$.

Given $\epsilon>0$, there is a set $D=\{|x| \leq a,|y| \leq b\}$, with $0<a, b<\epsilon$, such that 
(1) $\tilde{\xi}_{n}^{\prime} \cap D=\{y=\zeta(x),|x| \leq a\}$ and

(2) $\tilde{\mathscr{S}} \cap D=\{y=f(x),|x| \leq a\}$.

We can easily construct a homeomorphism $\tilde{h}: D \rightarrow D$ satisfying

(1) $\tilde{h}(\tilde{\mathscr{S}} \cap D)=\tilde{\xi}_{n}^{\prime} \cap D$ and

(2) $\tilde{h}(x, y)=(x, y)$ if $|y|=b$,

which is extended as a homeomorphism between two neighborhoods of $E$ by setting $\tilde{h}=$ id outside $D$, where id stands for the identity map. Then the map $h=\pi \circ \tilde{h} \circ \pi^{-1}$ defines a homeomorphism between two neighborhoods $U_{1}$ and $U_{2}$ of $0 \in \mathbb{C}^{2}$ such that:

(1) $h\left(\mathscr{S} \cap U_{1}\right)=\xi_{n}^{\prime} \cap U_{2}$;

(2) $h(\pi(D))=\pi(D)$ and $h=$ id outside $\pi(D)$.

Thus, the map $\mathfrak{h}=h \circ \Phi_{n}$ is a topological equivalence between $\xi_{n}$ and $\xi_{n}^{\prime}$. Moreover, since $\mathfrak{h}$ coincides with $\Phi_{n}$ outside $\pi(D)$, a similar construction as above can be successively made in an infinitesimal neighborhood of each irreducible component of $\mathscr{C}$ in order to obtain, for $n$ large enough, a topological equivalence $\mathfrak{h}$ between $\mathscr{C}$ and $\mathscr{C}^{\prime}$.

We close this section by establishing a kind of "factorization" theorem for a real parametrization of an irreducible complex curve. In more precise terms, suppose that $\xi$ is an irreducible curve at $\left(\mathbb{C}^{2}, 0\right)$ defined by the formal equation $F(u, v)=0$. Let

$$
\Gamma=(f(x, y), g(x, y))
$$

be a formal parametrized real surface at $\left(\mathbb{C}^{2}, 0\right)$ whose "image" is contained in $\xi$, that is, such that $F(f, g)=0$. Then, Lemma 7.3 asserts that $\Gamma$ is a formal real reparametrization of a Puiseux parametrization of $\xi$. This result and its Corollary 7.4 will be important in the proof of Theorem I.

Lemma 7.3. Let $F$ be an irreducible element in $A=\mathbb{C}[[x, y]]$ and let $\sigma \in \mathbb{C}[[z]], n \in \mathbb{N}$ be such that $\left(z^{n}, \sigma(z)\right)$ is a Puiseux parametrization for the formal curve $F=0$. Let $f, g \in A$ be such that $F(f, g)=0$. Then there exists a series $\psi \in A$ such that

$$
(f, g)=\left(\psi^{n}, \sigma(\psi)\right) .
$$

Proof. We will first show that it is sufficient to prove that $f$ has an $n^{\text {th }}$ root in $A$. Suppose that there exists $\phi \in A$ such that $\phi^{n}=f$. By Puiseux's Theorem we have that

$$
F\left(t^{n}, y\right)=U \prod_{\xi^{n}=1}(y-\sigma(\xi t))
$$

where $U$ is a unit in $\mathbb{C}[[t, y]]$. Since $F\left(\phi^{n}, g\right)=F(f, g)=0$, we conclude from equation (7.2) that $g=\sigma(\xi \phi)$ for some $\xi$ such that $\xi^{n}=1$. Therefore it suffices to take $\psi=\xi \phi$.

Let us prove that $f$ has an $n^{\text {th }}$ root in $A$. We exclude the trivial case $n=1$ and suppose by contradiction that $f$ has no $n^{\text {th }}$ root in $A$. Denote by $Q$ the field of fractions of $A$. At first we will show that, without loss of generality, we can assume that the polynomial $z^{n}-f$ is irreducible in $Q[z]$. Let $d \in \mathbb{N}$ be the greatest divisor of $n$ such that $f$ has a $d^{\text {th }}$ root in $A$. Then there exists $\tilde{f} \in A$ such that $f=\tilde{f}^{d}$. Since $F\left(\tilde{f}^{d}, g\right)=0$, for some irreducible factor $\tilde{F}$ of $F\left(x^{d}, y\right)$ we have $\tilde{F}(\tilde{f}, g)=0$. If we set $\tilde{n}=\frac{n}{d}$, since $f=\tilde{f}^{d}$ has no $n^{\text {th }}$ root in $A$, we have that $\tilde{f}$ has no $\tilde{n}^{\text {th }}$ root in $A$. Therefore, we have that $\tilde{F}, \tilde{f}$ and $g$ satisfy the hypothesis of the lemma and $\tilde{f}$ has no $\tilde{n}^{\text {th }}$ root in $A$. Moreover, from the maximality of $d$ we see that, for any divisor $k \neq 1$ of $\tilde{n}$, the series $\tilde{f}$ has no $k^{\text {th }}$ root in 
$A$. Thus, without loss of generality we can assume that $f$ has no $k^{\text {th }}$ root in $A$ for any divisor $k \neq 1$ of $n$. This implies that, for any divisor $k \neq 1$ of $n$, the element $f$ has no $k^{\text {th }}$ root in the field $Q$ of fractions of $A$. From this we conclude that the polynomial $z^{n}-f$ is irreducible in $Q[z]$ (see, for instance, [5, Ch. VI.9]).

Given any $h \in A$, define $h^{*}(t, x)=h(x, t x)$. If we write $h=\sum_{j=0}^{\infty} h_{j}$, where $h_{j}$ is the homogeneous polynomial of degree $j$ in $\mathbb{C}[x, y]$, we obtain that

$$
h^{*}(t, x)=\sum_{j=0}^{\infty} h_{j}(1, t) x^{j} .
$$

Notice that $h_{j}(1, t)$ is a polynomial of degree at most $j$, so that the map $h \mapsto h^{*}$ defines an isomorphism from $A$ into a ring $A^{*}$ contained in the ring $K[[x]]$ of formal power series with coefficients in the field $K=\mathbb{C}(t)$ of complex rational functions in the variable $t$. In particular, if $f=\sum_{j \geq \nu} f_{j}$ with $f_{\nu} \neq 0$, we obtain that

$$
f^{*}=\sum_{j \geq \nu}^{\infty} f_{j}(1, t) x^{j}, \quad f_{\nu}(1, t) \neq 0 .
$$

If $\bar{K}$ is the algebraic closure of $K$, we know that the series $f^{*}$ has an $n^{\text {th }} \operatorname{root} \psi$ in $\bar{K}[[x]]$. Then $\psi$ is a root of the polynomial $z^{n}-f^{*} \in A^{*}[z]$. Since the polynomial $z^{n}-f$ is irreducible in $Q[z]$, it follows that $n$ is the minimum degree of a nonzero polynomial in $A^{*}[z]$ having $\psi$ as a root. Since $\mathbb{C} \subset \bar{K}$, we will consider $F$ as an element in $\bar{K}[[x, y]]$. Then, since $F\left(f^{*}, g^{*}\right)=0$ and $f^{*}=\psi^{n}$, it follows from Puiseux's Theorem in $\bar{K}[[x, y]]$ that $g^{*}=\sigma(\xi \psi)$ for some $\xi, \xi^{n}=1$. Without loss of generality we can assume that $\xi=1$. If we do the substitution $\psi^{n}=f^{*}$ in the equation $g^{*}=\sigma(\psi)$, for some series $\sigma_{1}, \ldots, \sigma_{n-1} \in \mathbb{C}[[z]]$ we obtain an equation of the form

$$
-g^{*}+\sigma_{1}\left(f^{*}\right) \psi+\ldots+\sigma_{n-1}\left(f^{*}\right) \psi^{n-1}=0 .
$$

Thus, since $\sigma_{j}\left(f^{*}\right)=\left(\sigma_{j}(f)\right)^{*} \in A^{*}$, we have that $\psi$ is a root of the polynomial

$$
P=-g^{*}+\sigma_{1}\left(f^{*}\right) z+\ldots+\sigma_{n-1}\left(f^{*}\right) z^{n-1} \in A^{*}[z] .
$$

Then, since $n$ is the minimum degree of a polynomial in $A^{*}[z]$ vanishing on $\psi$, we conclude that $P=0$. Then $g=0$ and consequently we have the equation $F(f, 0)=0$. Therefore, if we express

$$
F(x, y)=\sum_{j \geq 0} s_{j}(x) y^{j}
$$

with $s_{j}(x) \in \mathbb{C}[[x]]$, we obtain that $s_{0}(f)=0$. This implies that $s_{0}=0$, because $f \neq 0$. Then, since $F$ is irreducible, we have that $F=U y$ for some unit $U \in A$. But this implies that $n=1$, which is a contradiction.

Let $F$ be an irreducible element in $\mathbb{C}[[x, y]]$. We say that a formal parametrized complex curve

$$
\Gamma(z)=\sum_{j \in \mathbb{N}} a_{j} z^{j}, a_{j} \in \mathbb{C}^{2}
$$

is a complex parametrization of the curve $F=0$ if $\Gamma \neq 0$ and we have $F(\Gamma(z))=0$. We say that the complex parametrization $\Gamma$ is reducible if there exist another formal parametrized complex curve $\tilde{\Gamma}$ and an element $\varphi \in \mathbb{C}[[z]]]$ with $\operatorname{ord}(\varphi)>1$ such that 
$\Gamma(z)=\tilde{\Gamma}(\varphi(z))$. Otherwise we say that $\Gamma$ is an irreducible complex parametrization of $F=0$. As a consequence of Lemma 7.3 , we have:

Corollary 7.4. Let $F$ be an irreducible element in $\mathbb{C}[[x, y]]$ and let $\sigma \in \mathbb{C}[[z]], n \in \mathbb{N}$ be such that $\left(z^{n}, \sigma(z)\right)$ is a Puiseux parametrization for the formal curve $F=0$. Let $\Gamma$ be any irreducible complex parametrization of $F=0$. Then there exists a formal complex diffeomorphism $\varphi \in \mathbb{C}[[z]]$ such that

$$
\Gamma=\left(\varphi^{n}, \sigma(\varphi)\right)
$$

Proof. Let $\Gamma=(f, g)$, where $f, g \in \mathbb{C}[[z]]$. Since $F(f, g)=0$ and $(f, g)$ can be considered as a formal real surface, by Lemma 7.3 there exists $\psi \in \mathbb{C}[[x, y]]$ such that

$$
(f, g)=\left(\psi^{n}, \sigma(\psi)\right) .
$$

Since $\left(z^{n}, \sigma(z)\right)$ is a Puiseux parametrization for the curve $F=0$, this curve is different from the $y$-axis and therefore $f \neq 0$. Then $\psi \neq 0$ and, since

$$
\psi^{n}=f \in \mathbb{C}[[z]],
$$

we deduce that $\psi$ is in fact a non-null complex series: there exists $\varphi \in \mathbb{C}[[z]], \varphi \neq 0$ such that

Then we have that

$$
\psi(x, y)=\varphi(x+i y) .
$$

$$
\Gamma=\left(\varphi^{n}, \sigma(\varphi)\right)
$$

and, since $\Gamma$ is an irreducible complex parametrization, we conclude that $\operatorname{ord}(\varphi)=1$ and therefore $\varphi$ is a formal complex diffeomorphism.

\section{8. $C^{\infty}$ EQUIVALENCES OF FOLIATIONS AND EQUISINGULARITY OF THE SET OF SEPARATRICES}

This section is devoted to prove Theorem 专.

Theorem 8.1. Let $\Phi$ be a $C^{\infty}$ equivalence between two germs $\mathcal{F}$ and $\mathcal{F}^{\prime}$ of singular holomorphic foliations at $\left(\mathbb{C}^{2}, 0\right)$. Let $S$ be a formal separatrix of $\mathcal{F}$ and let $S^{\prime}=\Phi_{*}(S)$ be the corresponding separatrix of $\mathcal{F}^{\prime}$ according to Theorem 6.1. Then $\hat{\Phi}$ is a formal real equivalence between $S$ and $S^{\prime}$.

Proof. Take coordinates $(z, w)$ in $\left(\mathbb{C}^{2}, 0\right)$ an suppose that $S^{\prime}$ is defined by a formal equation $F=0$, where $F \in \mathbb{C}[[z, w]]$ is irreducible. As a first step, considering $S$ as a formal real surface, we will prove that $F \circ \hat{\Phi} \circ S=0$. Since this is obvious if $S$ is convergent, we assume that $S$ is a weak separatrix. Let $\pi:(M, E) \rightarrow\left(\mathbb{C}^{2}, 0\right)$ be the reduction of singularities of $\mathcal{F}$. Then, the strict transform $\tilde{S}=\pi^{*} S$ is the weak separatrix of a saddle-node singularity at some $p \in E$. Let $(u, v)$ be local holomorphic coordinates at $p \in E$ such that:

(1) $p \simeq(0,0)$;

(2) $E$ is given by $\{u=0\}$.

There exists a formal series $\hat{s}(u)=\sum_{j=1}^{\infty} c_{j} u^{j}$ such that $\tilde{S}$ is given by $v=\hat{s}(u)$, hence the separatrix $S(u)=\pi(u, \hat{s}(u))$ is parametrized as a real surface by

$$
S(x, y)=\pi(x+i y, \hat{s}(x+i y)) .
$$


In order to prove that $F \circ \hat{\Phi} \circ S=0$ it is sufficient to show that, if $\alpha, \beta \in \mathbb{R}^{*}$ are arbitrarily chosen, then the series

$$
f(t):=F \circ \hat{\Phi} \circ S(\alpha t, \beta t)
$$

is null. If we set $\eta=\alpha+i \beta$, the series $f$ can be expressed as

$$
f=F \circ \hat{\Phi} \circ S(\eta t) \text {. }
$$

As we have seen in Example 5.7, we know that $S(\eta t)$ is the Taylor series of a characteristic curve $\gamma$ of $\mathcal{F}$ asymptotic to $S$. Then, since $\gamma^{\prime}:=\Phi(\gamma)$ is a characteristic curve of $\mathcal{F}^{\prime}$ asymptotic to $S^{\prime}$, we deduce that $\hat{\gamma}^{\prime}=\hat{\Phi} \circ S(\eta t)$ is a complex formal parametrization of $S^{\prime}$ and therefore

$$
f=F \circ \hat{\Phi} \circ S(\eta t)=F \circ \hat{\gamma}^{\prime}=0 .
$$

Without loss of generality we can assume that both curves $S$ and $S^{\prime}$ are tangent to the $z$ axis, which implies the following properties:

(1) $S$ has a Puiseux parametrization $\left(T^{n}, \sigma(T)\right)$, where $n$ is the multiplicity of the curve $S$ and $\sigma \in \mathbb{C}[[T]]$, ord $(\sigma)>n$;

(2) $S^{\prime}$ has a Puiseux parametrization $\left(T^{n^{\prime}}, \sigma^{\prime}(T)\right)$, where $n^{\prime}$ is the multiplicity of the curve $S^{\prime}$ and $\sigma^{\prime} \in \mathbb{C}[[T]]$, ord $\left(\sigma^{\prime}\right)>n^{\prime}$.

By Proposition 6.3 and without loss of generality — the other case is similar — we can assume that $\hat{\Phi}(z, w)$ has a complex linear part

$$
\hat{\Phi}_{1}(z, w)=(a z+b w, c z+d w), a d-b c \neq 0 .
$$

Suppose that

$$
S(u)=\left(\sum_{j \geq \bar{n}} \alpha_{j} u^{j}, \sum_{j \geq \bar{n}} \beta_{j} u^{j}\right),\left(\alpha_{\bar{n}}, \beta_{\bar{n}}\right) \neq(0,0) .
$$

Since it is an irreducible parametrization, by Corollary 7.4 there exists a formal diffeomorphism $\varphi \in \mathbb{C}[[u]]$ such that $S=\left(\varphi^{n}, \sigma(\varphi)\right)$, hence $\bar{n}=n$ and $\beta_{n}=0$. Therefore, from (8.1) and 8.2) above we have that the initial part of $\hat{\Phi} \circ S$ is complex and is given by

$$
(\hat{\Phi} \circ S)_{1}=\left(a \alpha_{n} u^{n}, c \alpha_{n} u^{n}\right) \text {. }
$$

Since $S^{\prime}$ is tangent to the $z$ axis, we have that $F$ has an initial part of the form $F_{N}=\mu y^{N}$, $\mu \neq 0, N \in \mathbb{N}$. Then, since $F \circ \hat{\Phi} \circ S=0$ implies $F_{N} \circ(\hat{\Phi} \circ S)_{1}=0$, we deduce that $c=0$ and consequently $a \neq 0$. By Lemma 7.3 , since $F(\hat{\Phi} \circ S)=0$, there exists $\psi \in \mathbb{C}[[x, y]]$ such that

$$
\hat{\Phi} \circ S(x+i y)=\left(\psi^{n^{\prime}}, \sigma^{\prime}(\psi)\right) .
$$

Then, since $(\hat{\Phi} \circ S)_{1}=\left(a \alpha_{n} u^{n}, 0\right)$, the initial part $\psi_{\nu}, \nu \in \mathbb{N}$ of $\psi$ satisfies the equality $a \alpha_{n} u^{n}=\psi_{\nu}^{n^{\prime}}$. Then $n=n^{\prime} \nu$ and therefore $n^{\prime} \leq n$. A similar argument using the inverse diffeomorphism $\Phi^{-1}$ allows us to conclude that $n=n^{\prime}, \nu=1$ and, consequently, $\psi$ has a linear part of the form $\sqrt[n]{a \alpha_{n}}(x+i y)$. Thus $\psi$ is a formal real diffeomorphism and therefore $\hat{\Phi} \circ S$ is a formal real reparametrization of $S^{\prime}$.

Proof of Theorem [4. It is a direct consequence of Theorems 8.1 and 7.2 . 


\section{The Proof of Theorem II}

Let $\mathcal{F}$ and $\mathcal{F}^{\prime}$ be germs of foliations, equivalent by a germ of $C^{\infty}$ diffeomorphism

$$
\Phi:\left(\mathbb{C}^{2}, 0\right) \rightarrow\left(\mathbb{C}^{2}, 0\right)
$$

Let $S \in \operatorname{Sep}(\mathcal{F})$ be a branch of separatrix and $S^{\prime}=\Phi_{*} S \in \operatorname{Sep}\left(\mathcal{F}^{\prime}\right)$ be the corresponding separatrix given by Theorem 1 . This result also asserts that, if $\mathscr{S}=\cup_{i=1}^{k} S_{i}$ is a reduced curve formed by the union of a finite number of branches in $\operatorname{Sep}(\mathcal{F})$, we set $\mathscr{S}^{\prime}=\Phi_{*} \mathscr{S}=$ $\cup_{i=1}^{k} \Phi_{*} S_{i}$, then $\mathscr{S}$ and $\mathscr{S}^{\prime}$ are equisingular. As a consequence, we have that $S \in \operatorname{Iso}(\mathcal{F})$ if and only if $S^{\prime} \in \operatorname{Iso}\left(\mathcal{F}^{\prime}\right)$ and $S \in \operatorname{Dic}(\mathcal{F})$ if and only if $S^{\prime} \in \operatorname{Dic}\left(\mathcal{F}^{\prime}\right)$. We have clearly the following more general fact:

Proposition 9.1. $\mathcal{F}$ and $\mathcal{F}^{\prime}$ are $\mathcal{S}$-equisingular.

Suppose now that $\hat{F}$ is a balanced equation of separatrices for $\mathcal{F}$, whose divisor is as in (3.1). We define $\hat{F}^{\prime}=\Phi_{*} \hat{F}$ as any formal meromorphic function corresponding to the following divisor

$$
\left(\hat{F}^{\prime}\right)_{0}-\left(\hat{F}^{\prime}\right)_{\infty}=\sum_{S \in \operatorname{Iso}(\mathcal{F})}\left(S^{\prime}\right)+\sum_{S \in \operatorname{Dic}(\mathcal{F})} a_{S}\left(S^{\prime}\right) .
$$

Lemma 9.2. Let $\mathcal{F}$ and $\mathcal{F}^{\prime}$ be germs of foliations, equivalent by a germ of $C^{\infty}$ diffeomorphism $\Phi:\left(\mathbb{C}^{2}, 0\right) \rightarrow\left(\mathbb{C}^{2}, 0\right)$. Let $S \in \operatorname{Dic}(\mathcal{F})$ and $S^{\prime}=\Phi_{*} S \in \operatorname{Dic}\left(\mathcal{F}^{\prime}\right)$ be corresponding separatrices, attached to dicritical components $D$ and $D^{\prime}$ of the desingularizations of $\mathcal{F}$ and $\mathcal{F}^{\prime}$. Then $\operatorname{Val}(D)=\operatorname{Val}\left(D^{\prime}\right)$.

Proof. This is a consequence of Proposition 9.1 and of the following fact: if $\pi:(M, E) \rightarrow$ $\left(\mathbb{C}^{2}, 0\right)$ is the reduction of singularities for $\mathcal{F}$ and $\mathcal{D} \subset E$ is the union of all dicritical components, then each connected component of $E \backslash \mathcal{D}$ carries a separatrix of $\mathcal{F}$ (see $[$, Prop. 4]).

This lemma allows us to prove the following:

Proposition 9.3. Let $\mathcal{F}$ and $\mathcal{F}^{\prime}$ be germs of foliations, equivalent by a germ of $C^{\infty}$ diffeomorphism $\Phi:\left(\mathbb{C}^{2}, 0\right) \rightarrow\left(\mathbb{C}^{2}, 0\right)$. If $\hat{F}$ is a balanced equations of separatrices for $\mathcal{F}$, then $\hat{F}^{\prime}=\Phi_{*} \hat{F}$ is a balanced equation of separatrices for $\mathcal{F}^{\prime}$. Besides, $\nu_{0}(\hat{F})=\nu_{0}\left(\hat{F}^{\prime}\right)$.

Proof. The isolated separatrices of $\mathcal{F}$ and $\mathcal{F}^{\prime}$ are in correspondence by $\Phi$, so that they appear in the zero divisor of both balanced equations with coefficient 1 . Similarly, there is a correspondence between dicritical separatrices of $\mathcal{F}$ and $\mathcal{F}^{\prime}$, which, by Lemma 9.2, are attached to dicritical components having the same valences. Therefore, $\hat{F}^{\prime}$ is a balanced equation for $\mathcal{F}^{\prime}$. Finally, the equality on the algebraic multiplicities follows from the equisingularity property given by Theorem $\mathbb{E}$.

The final ingredient for the proof of Theorem [1] is the following result of [9]:

Theorem 9.4. Let $\mathcal{F}$ and $\mathcal{F}^{\prime}$ be germs at $\left(\mathbb{C}^{n}, 0\right)$ of $C^{1}$ equivalent one dimensional foliations. Then $\nu_{0}(\mathcal{F})=\nu_{0}\left(\mathcal{F}^{\prime}\right)$.

This enables to prove the following:

Proposition 9.5. The tangency excess $\tau_{0}(\mathcal{F})$ is a $C^{\infty}$ invariant. 
Proof. Let $\Phi$ be a $C^{\infty}$ equivalence between $\mathcal{F}$ and $\mathcal{F}^{\prime}$. We have $\nu_{0}(\mathcal{F})=\nu_{0}\left(\mathcal{F}^{\prime}\right)$ by the previous theorem. Moreover, Proposition 9.3 gives that if $\hat{F}$ be a balanced equation of separatrices for $\mathcal{F}$, then $\hat{F}^{\prime}=\Phi_{*} \hat{F}$ is a balanced equation of separatrices for $\mathcal{F}^{\prime}$ and $\nu_{0}(\hat{F})=\nu_{0}\left(\hat{F}^{\prime}\right)$. The result then follows from Proposition 3.6.

We are now ready to complete the proof of Theorem II:

Proof of Theorem II Let $\mathcal{F}$ and $\mathcal{F}^{\prime}$ be $C^{\infty}$ equivalent foliations. Being $\mathcal{F}$ of second type, it holds $\tau_{0}(\mathcal{F})=0$. Consequently, by Proposition 9.5, $\tau_{0}\left(\mathcal{F}^{\prime}\right)=0$ and $\mathcal{F}^{\prime}$ is also of second type. Hence, both $\mathcal{F}$ and $\mathcal{F}^{\prime}$ are $\mathcal{S}$-desingularizable by Proposition 3.2. The proof is accomplished by using the fact that $C^{\infty}$ equivalent foliations are $\mathcal{S}$-equisingular (Proposition 9.1).

\section{REFERENCES}

[1] C. Camacho, A. Lins Neto, and P. Sad. Topological invariants and equidesingularization for holomorphic vector fields. J. Differential Geom., 20(1):143-174, 1984.

[2] F. Cano, N. Corral, and R. Mol. Local polar invariants for plane singular foliations. arXiv:1508.06882, 2015.

[3] Y. Genzmer. Rigidity for dicritical germ of foliation in $\mathbb{C}^{2}$. Int. Math. Res. Not. IMRN, (19):1073-7928, 2007.

[4] Y. Genzmer and R. Mol. The poincaré problem in the dicritical case. arXiv:1508.06882, 2016.

[5] Serge Lang. Algebra, volume 211 of Graduate Texts in Mathematics. Springer-Verlag, New York, third edition, 2002.

[6] J.-F. Mattei and E. Salem. Modules formels locaux de feuilletages holomorphes. arXiv:math/0402256, 2004.

[7] R. Mol. Meromorphic first integrals: some extension results. Tohoku Math. J. (2), 54(1):85-104, 2002.

[8] Rudy Rosas. The differentiable-invariance of the algebraic multiplicity of a holomorphic vector field. J. Differential Geom., 83(2):337-376, 2009.

[9] Rudy Rosas. The $C^{1}$ invariance of the algebraic multiplicity of a holomorphic vector field. Ann. Inst. Fourier (Grenoble), 60(6):2115-2135, 2010.

[10] Rudy Rosas. Bilipchitz invariants for holomorphic foliations. Int. Math. Res. Not. IMRN, (11):3425$3472,2016$.

[11] A. Seidenberg. Reduction of singularities of the differential equation $A d y=B d x$. Amer. J. Math., 90:248-269, 1968.

[12] Oscar Zariski. On the Topology of Algebroid Singularities. Amer. J. Math., 54(3):453-465, 1932.

Rogério Mol

Departamento de Matemática

Universidade Federal de Minas Gerais

Av. Antônio Carlos, 6627 C.P. 702

30123-970 - Belo Horizonte - MG, Brasil

rsmol@mat.ufmg.br

Rudy Rosas

Pontificia Universidad Católica del Perú

Av. Universitaria 1801

Lima, Peru

rudy.rosas@pucp.edu.pe 